\title{
A high-precision miniaturized rotating coil transducer for magnetic measurements
}

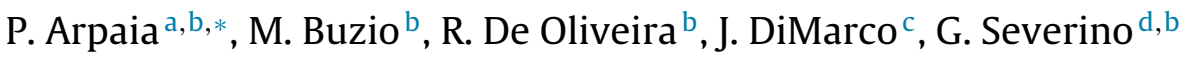 \\ a Department of Electrical Engineering and Information Technology, University of Naples Federico II, Naples, Italy \\ b Technology Department, European Organization for Nuclear Research (CERN), Geneva, Switzerland \\ ${ }^{\text {c } T e c h n i c a l ~ D i v i s i o n, ~ F e r m i l a b, ~ B a t a v i a, ~ I L, ~ U S A ~}$ \\ ${ }^{\mathrm{d}}$ Department of Engineering, University of Sannio, Benevento, Italy
}

\section{A R T I C L E I N F O}

\section{Article history:}

Received 28 September 2017

Received in revised form 12 January 2018

Accepted 31 January 2018

Available online 8 February 2018

\section{Keywords:}

84.32.Hh/coils, induction

85.75.Ss/sensors magnetic field

06.20.fb/calibration

07.55.Ge/measurement of magnetic field

41.20.-q/magnetic field in

electromagnetism

\begin{abstract}
A B S T R A C T
A miniaturized Printed Circuit Board (PCB) sensing coil, jointly developed by CERN and Fermilab for measuring the field of small-gap (less than $10 \mathrm{~mm}$ ) accelerator magnets, is illustrated. A sensing coil array, with a scheme for compensating the main field when measuring the harmonic error components, hosted on a synthetic sapphire-based transducer, is presented. Key innovating features are (i) very-small size, both for the sensing coil array (thickness of $1.380 \mathrm{~mm}$ ) and for the transducer (overall diameter of $7.350 \mathrm{~mm}$ ), (ii) metrological performance, namely accuracy (more than five times better than state of the art), and 1-sigma repeatability (ten times better on harmonics with amplitude less than 100 ppm), and (iii) manufacturing technology of both the coil array (13 double layers aligned within $10 \mu \mathrm{m}$ ), and the sapphire support (concentricity, the most important uncertainty source for rotating coils, $3 \mu \mathrm{m}$ of uncertainty, namely one order of magnitude better than fiberglass support). After stating the measurement problem, the design of the transducer and a case study of a two-layer PCB sensor array are illustrated. Then, the prototyping and quality control of both the sensor and the transducer are discussed. Furthermore, the calibration and the results obtained with a prototype setup at Fermilab are presented. Finally, in the appendix, the theory of the rotating coil, the sensor geometry, and the harmonic compensation are briefly reviewed for the reader easiness.
\end{abstract}

Published by Elsevier B.V.

\section{Introduction}

Sensing induction coils have been used in several different applications [1], such as food quality control [2], high-frequency drive thin-film micro-magnetic devices [3], portable navigation systems [4], material characterization [5], magnetic tracking systems [6], space research [7], and proximity sensors and detectors [8].

A research and development field, where sensing induction coils received a significant technological impulse, has been beam-line magnets of particle accelerators. In fact, magnet quality depends so critically upon uncertainty sources, such as mechanical and assembly tolerances, and material properties, that they must be fully characterized experimentally before installation $[9,10]$. The most important properties to be measured are strength and direction of the main magnetic field component, the field errors, usually

\footnotetext{
* Corresponding author at: Department of Electrical Engineering and Information Technology, University of Naples Federico II, Naples, Italy

E-mail addresses: pasquale.arpaia@unina.it (P. Arpaia), giordana.severino@cern.ch (G. Severino).
}

expressed in terms of high-order harmonics [11], and the offset of the magnetic center in case of multipoles [12,13]. Different experimental techniques exist to perform a full magnet characterization. One of the preeminent and most reliable techniques is the rotating or harmonic coil method [12,14-18]. This method, based on an assembly of induction coils rotating inside the magnet bore, is generally capable of providing field error measurements with a repeatability of a few ppm [19] [20]. However, especially in the case of axially-long coils and small diameters, mechanical imperfections and vibrations can induce spurious harmonics that compromise the quality of the final result [21].

Design, fabrication, and testing of coil assemblies able to minimize vibration and geometry nonideality, while maintaining sufficient sensitivity, even in the case of extreme length/diameter aspect ratios, turns out to be a challenging issue compared to bore sizes bigger that $10 \mathrm{~mm}$ [22-24]. At CERN, a prototype of PCB coil array [25], with diameter of $8 \mathrm{~mm}$ and tangential windings geometry, was previously developed to gain experience with PCB coil technology. It was optimized to have the largest number of winding turns in the smallest possible space. Initially, this sensor was 
used to test the relatively weak permanent magnet quadrupoles of the new accelerator Linac4 at CERN (up to $4 \mathrm{Tm} / \mathrm{m}$ of integrated field gradient) [26]. Then, this sensor proved to be up to the task of characterizing the magnets of the Swiss XFEL, an advanced synchrotron light source currently under construction at the Paul Scherrer Institute (PSI), Villigen, CH [25]. However, its capability to reject spurious harmonics arising from mechanical imperfections is not fully satisfactory. Hence, a new design able to tackle this issue is required.

In the context of the European project PACMAN [27] at CERN, the main driver to develop small-diameter rotating coils has been the need to test the field quality of prototype final focusing quadrupoles for the CLIC project [28]. The prototype of the final-focus magnet QD0 is a compact hybrid magnet, with four permanent magnet inserts, each composed by 4 blocks glued together, and classical electro-magnetic coils. It has a very-small size of the bore, with a diameter of only $8.25 \mathrm{~mm}$, necessary to get the very-high gradient of $200 \mathrm{~T} / \mathrm{m}$ (about $70 \mathrm{Tm} / \mathrm{m}$ integrated gradient).

This kind of last-generation magnets, with very small bore and very high field quality, poses metrological challenges to PCB sensor design and manufacturing. Promising performance was obtained at Fermilab by a radial array of coils [29], capable of improving stateof-the-art separated coils by a unique PCB compensation. This coil array was exploited successfully for the cryogenic measurements of the LARP high-gradient HQ magnet program [29]. However, this solution is not applicable yet to diameter scales less than of $10 \mathrm{~mm}$.

In this paper, an enhancement of the coil sensor based on PCB technology, originally developed at Fermilab [29,30] and further at CERN, is proposed. An innovative, ultra-thin (less than $2 \mathrm{~mm}$ ) sensing coil array, with a compensation scheme for the main field components, and a synthetic sapphire support of the transducer, jointly developed by CERN and Fermilab, are presented. The design aims to minimize the impact of the finite thickness on the measurement accuracy, in particular of the harmonic field error components. In Section 2, after stating the measurement problem, the design of the transducer and a case study of a two-layer PCB sensor array are illustrated. In Sections 3 and 4, the prototyping and quality control of the sensor and of the transducer, respectively, specifically optimized for such a small diameter, are discussed. In Section 5 , the calibration and the results obtained with a prototype setup at Fermilab are presented. Finally, in the appendix, the theory of the rotating coil, the sensor geometry, and the harmonic compensation concepts are briefly reviewed.

\section{Design of a miniaturized $\mathrm{PCB}$ rotating coil}

\subsection{Measurement problem}

A transducer based on a rotating coil array is required for testing quadrupole magnets with very small size of the magnet bore (diameter less than $10 \mathrm{~mm}$ ), and very high field quality (undesired harmonic content with amplitude less than $100 \mathrm{ppm}$ of the nominal quadrupole). The miniaturization should not affect both the measurement accuracy and the sensor sensitivity, in particular for the harmonic field error components, in line of principle reduced by downgrading coil area and thickness.

A further key improvement concerns the compensation of the dipole and quadrupole components, in the order of at least two orders of magnitude with respect to traditional designs.

Therefore, main aim is to design an array of ideally identical coils to be connected to provide (i) an absolute output (unbucked, absolute signal), with the largest sensitivity possible to the quadrupole component (expressed as the coefficient $\kappa_{2}$, see Appendix A.2), (ii) a compensated channel (dipole-bucked, DB signal), with the smallest sensitivity to dipole ( $\kappa_{1}$ in Appendix A.2) and highest to quadrupole

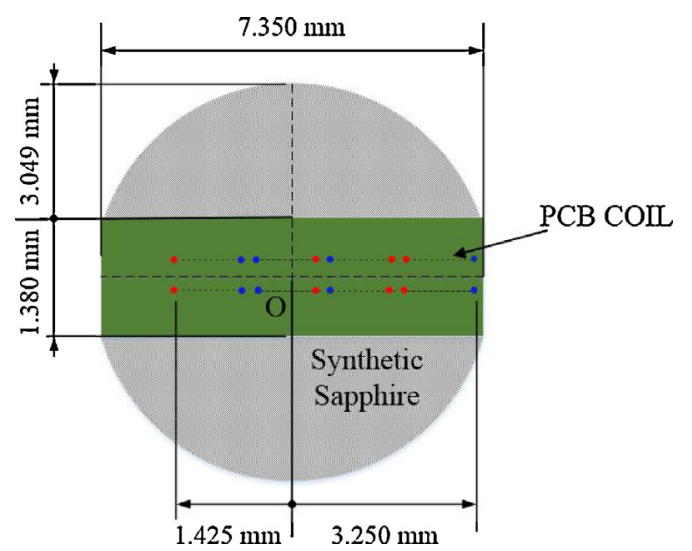

Fig. 1. $P C B$ rotating coil section, with equivalent tracks.

Table 1

Output signals with connections of the proposed PCB coil array.

\begin{tabular}{lll}
\hline Output & Type & Connections \\
\hline UB & Un-bucked (absolute) & $(\# 1)$ \\
DB & Dipole-bucked & $(\# 1-3)$ \\
DQB & Dipole- and quadrupole-bucked & $(\# 1-3+4-2)$ \\
\hline
\end{tabular}

$\left(\kappa_{2}\right)$ possible, and (iii) a further compensated channel (dipole-and quadrupole-bucked, DQB signal), with the smallest $\kappa_{1}$ and $\kappa_{2}$ and highest sensitivity to sextupole component ( $\kappa_{3}$ in Appendix A.2).

\subsection{Transducer design}

The design aims at minimizing the impact of the coil thickness on the measurement accuracy, in particular for the harmonic field error components, while preserving adequate sensitivity. The coil array is made to be mounted on the diameter of a cylindrical shaft, at a position precisely defined by two matching pin holes. The section is illustrated in Fig. 1, with four equal rectangular coils with hardwired compensation.

Signals arising from multiple series combinations of the same coils, considered as voltage sources with a typical resistance of a few $\mathrm{k} \Omega$, can be acquired with differential input impedance of the order of $10 \mathrm{M} \Omega$.

The main features of this design can be summarized as follows:

- the quality of the compensation is determined mainly by the geometry of the tracks on the PCB, namely by the accuracy of the surface and the distance of the coils;

- the bucking factor will be less sensitive to vibrations if the PCB is mounted radially by minimizing transverse offset or tilt angle;

- sensitivity can be tuned during both the design (by choosing the total PCB thickness) and the assembly (by adjusting the transverse offset in the shaft);

- track density obtainable by the present optimized PCB design can be 2-3 times lower than conventional $30 \mu \mathrm{m}$ gauge wire [25], because in the same cross section 2-3 times less turns can be wound; however, high effective areas are only a secondary goal for high field magnets;

- a coil PCB fully-optimized in space results in very thick copper tracks with high resistance, in the range of several $\mathrm{k} \Omega$;

- the three obtainable output signals are highlighted in Table 1 with the corresponding connections.

\subsection{Case study: two-layer PCB}

Ideally, all the coils on the PCB lie on the same plane passing by the rotation axis. Therefore, according to Eq. (7) in Appendix A.2, all 


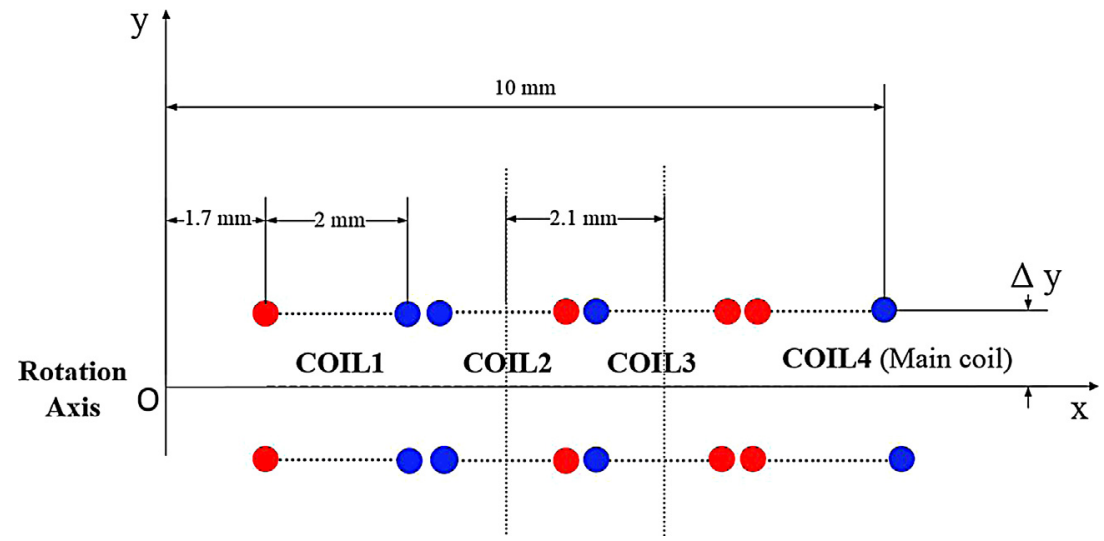

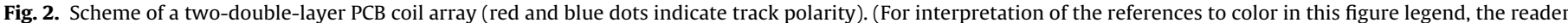
is referred to the web version of this article.)

sensitivity coefficients $\kappa_{n}$ are not null. However, the finite thickness of the PCB affects significantly the actual design performance. The impact of this non-ideality was studied numerically on a simplified model of the array, shown in Fig. 2 (red and blue dots indicate the polarity of the tracks).

This array includes four equal coils printed on two PCB layers, with a single turn on each layer. The width of the array is $10 \mathrm{~mm}$, with coils of $2 \mathrm{~mm}$ of width, spaced by $2.1 \mathrm{~mm}$. The normalized sensitivity coefficients of the quadrupole-bucked coil $\left(\kappa_{n}^{*}\right.$ in Appendix A.2) were calculated as a function of the distance $\Delta y$ between the two layers. $\Delta y$ was varied in the range $(0.4,4.0) \mathrm{mm}$, i.e. from $4 \%$ to $40 \%$ of the reference radius $R_{\text {ref. }}$. The reference radius is set equal to the abscissa of the outermost track. The sensitivity coefficients $\kappa_{n}$ are normalized with respect to the reference radius $\left(\kappa_{n}^{*}\right)$, in order to have all the dimension of an area $\left(\mathrm{m}^{2}\right)$ and a comparable order of magnitude, according to Eq. (9) in Appendix A.2.

The results in Fig. 3 show clearly that, as the thickness of the PCB increases, the compensated coil approaches the qualitative behavior of a tangential coil array, characterized by low-order sensitivity zeros or minima.

In particular, at the minimum thickness $(0.4 \mathrm{~mm})$, the magnitude of the calibration coefficients drops very slowly with $n$ and remains appreciably high even at $n=20$. On the other hand, at the maximum thickness $(4.0 \mathrm{~mm}), \kappa_{6}$ is nearly zero, and thus the corresponding measured harmonic will be under the noise floor. (The dodecapole, i.e. $n=6$, is the lowest harmonic allowed by the symmetry in a quadrupole; therefore, it is expected to be correctly predicted by computer simulation and so is often used to validate a measurement system). The lowest-order harmonics, most important in beam dynamics, are degraded only marginally when the thickness increases up to $1.9 \mathrm{~mm}$, the biggest loss being about $30 \%$ for $n=6$. Above $2.0 \mathrm{~mm}$, however, only the sextupole and octupole terms remain unaffected. The sensitivity to higher orders $n \geq 10$ increases greatly according to the PCB thickness, although these harmonics have an interest only in very special cases. As an example, the harmonic $n=11$ was used in superconducting dipoles of the CERN Large Hadron Collider to define a magnetic axis for alignment purposes [31].

In synthesis, this study confirms that the total thickness of the coil array must be below a certain fraction of the reference radius, about $10 \%$, in order to keep a satisfying sensitivity over a typical harmonic range of interest. This problem is exacerbated in the case of small magnet apertures, where the available gap is of the same order of magnitude than the thickness of a typical PCB. In the present case, a special manufacturing effort is needed to limit the thickness as much as possible. In practice, coils usually include many more layers, sometimes up to several dozens. If so, the total number of winding turns is equal for each of the four coil connected in compensation scheme, and the impact of higher thickness will be mitigated with respect to the results shown here. At any rate, the number of turns per layer should always be maximized taking into account the manufacturing limitations (module manufacturing) in order to keep the thickness at a reasonable level.

\section{Prototyping and quality control of the sensor}

\subsection{Sensor prototyping}

According to the above design principles, a prototype of a coil array for the CLIC quadrupoles was produced at CERN by the service Micro-Pattern Technologies (EP-DT-DD MPT). This CLIC application represents a rather extreme case for this kind of transducers, because the array is to be installed in a shaft with a diameter of only $7.45 \mathrm{~mm}$. The design parameters of the coils are reported in Table 2 and main manufacturing parameters of the PCB in Table 3.

Table 2

Design parameters of the coil prototype for CLIC quadrupoles (UB: un-bucked absolute-, DB: dipole-bucked, and DQB: dipole- and quadrupole-bucked).

\begin{tabular}{ll} 
Total thickness (nominal) & $1.38 \mathrm{~mm}$ \\
Total thickness (measured) & $1.28 \mathrm{~mm}$ \\
Total width & $7.35 \mathrm{~mm}$ \\
Coil width (outer tracks) & $0.55 \mathrm{~mm}$ \\
Coil width (inner tracks) & $1.15 \mathrm{~mm}$ \\
Coil inter-distance & $1.30 \mathrm{~mm}$ \\
Coil length & $0.152 \mathrm{~m}$ \\
Coil area & $0.01324 \mathrm{~m}^{2}$ \\
Layers & 26 \\
Layers with coils & 24 \\
Turns/layer & 4 \\
Total number of turns & 96 \\
Resistance UB & $2022 \Omega$ \\
Resistance DB & $4045 \Omega$ \\
Resistance DQB & $8089 \Omega$ \\
\hline
\end{tabular}

Table 3

PCB manufacturing parameters of a coil array prototype for CLIC quadrupoles (prepreg: pre-impregnated, FR-4: cured epoxy resin).

$\begin{array}{ll}\text { Distance between coil trace and cut } & 0.25 \mathrm{~mm} \\ \text { Via diameter } & <0.20 \mathrm{~mm} \\ \text { Internal pad width } & 0.25 \mathrm{~mm} \\ \text { External pad width } & 0.50 \mathrm{~mm} \\ \text { FR-4 sheet thickness } & 50 \mu \mathrm{m} \\ \text { Pre-preg sheet thickness } & 50 \mu \mathrm{m} \\ \text { Copper track thickness } & 5 \mu \mathrm{m} \\ \text { Copper track width } & 50 \mu \mathrm{m} \\ \text { Copper track distance } & 50 \mu \mathrm{m}\end{array}$



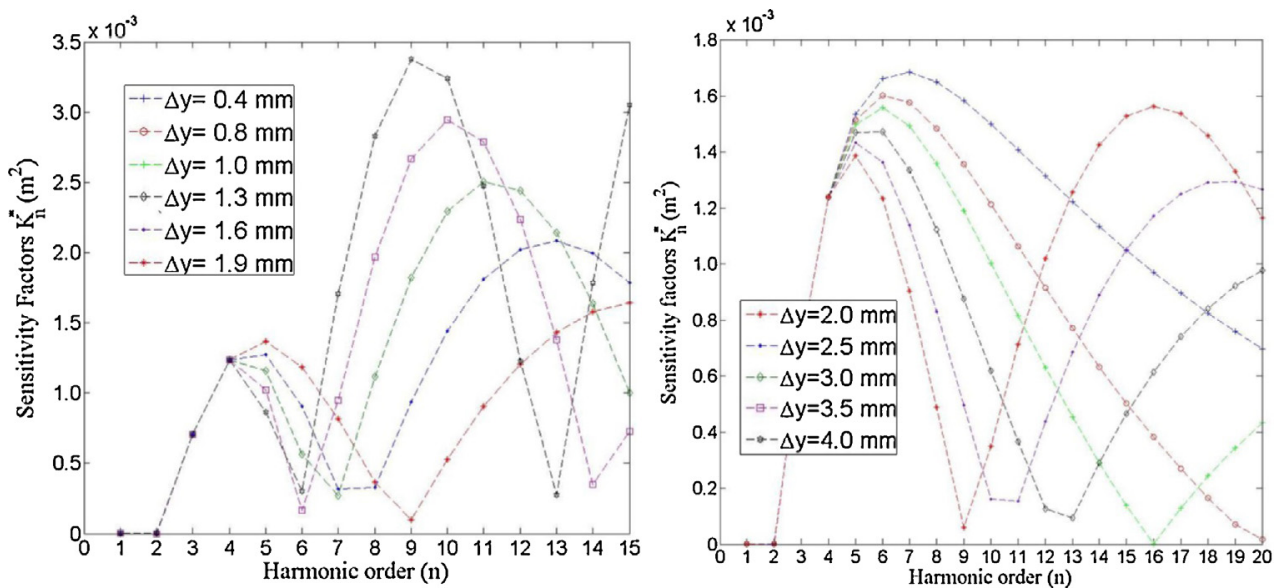

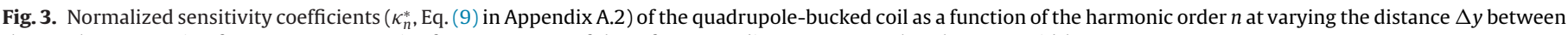
the two layers, varying from 0.4 to $4.0 \mathrm{~mm}$, i.e. from $4 \%$ to $40 \%$ of the reference radius $R_{\text {ref }}$, set equal to the array width.

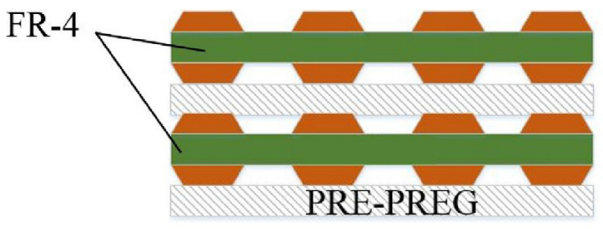

(A)

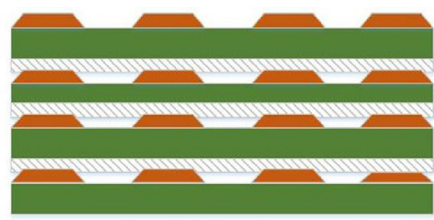

(B)

Fig. 4. Difference between PCB with a stack of (A) two double layers and (B) four single layers.

In particular, the PCB has 24 layers hosting the coils and further two layers used just for manufacturing reasons. In fact, the two external layers have bigger pads to guarantee the adhesion and to avoid vias damages; while the internal smaller pads make possible coils with smaller internal width (multiple turns). These parameters are unusual and could be achieved only by optimizing aggressively the production. For comparison, standard copper tracks are usually thicker than $10 \mu \mathrm{m}$, the cured epoxy resin (FR-4) and preimpregnated (pre-preg) are thicker than $80 \mu \mathrm{m}$, and the width and distance between copper tracks is at least $100 \mu \mathrm{m}$.

The layers were produced with the Laser Direct Imaging machine (LDI), exploiting a laser beam that exposes directly the dry film and so can image electric circuits without a photo-tool or a mask. The PCB design emphasizes the minimization of the total thickness. The double layers are made of FR- 4 with copper on both sides. They are insulated and held together by pre-preg layers, made of a mesh of fiberglass with uncured epoxy resin (Fig. 4A).

Single PCB layers, where the copper is only on one side, could also be used as an alternative to improve track alignment and overall thickness, as shown in Fig. 4B. In this case, the role of insulation is given alternately to the pre-preg and FR- 4 sheets. Alignment is completely done by hand using special tooling in a clean room, to avoid impurities between layers. The clean room is equipped with yellow lights to avoid undesired polymerization. The dry-film used is of negative type, which is sensitive to light under $540 \mathrm{~nm}$. Single layers are not commonly available off the shelf, although they can be obtained by removing the copper from one side by etching. Their use was initially foreseen in this project, but had to be postponed to a successive iteration.

\subsection{Sensor quality control}

The quality inspection and calibration of the first miniaturized PCB sensor prototype was carried out at Fermilab, where the PCB was assembled in a G-10 shaft produced in-house. The sen-

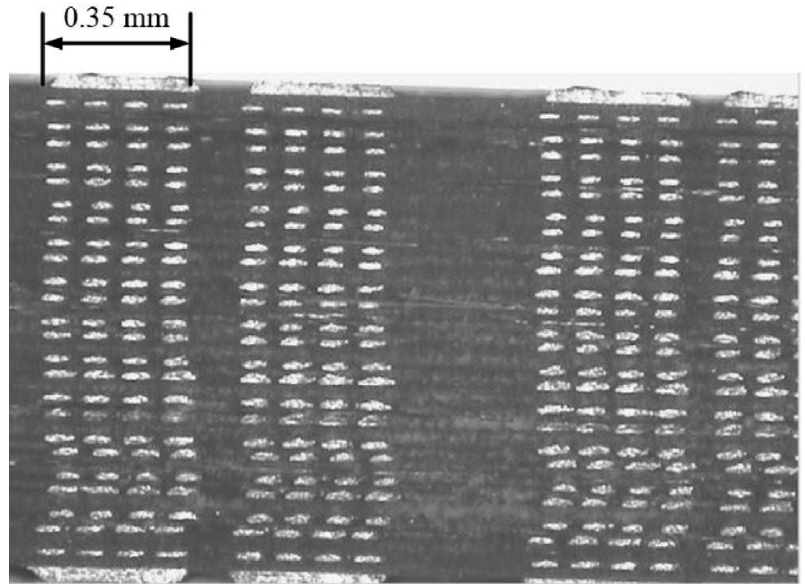

Fig. 5. Microscopic view of PCB cross-cut of finished PCB taken from coupon board, the part of the external frame (removed during the $\mathrm{PCB}$ cut phase) used to verify the manufacturing quality.

sors were inspected through a microscope to verify the quality of the manufacturing process, by paying particular attention to the final geometry of the coil turns (Fig. 5). The microscopic inspection showed that the 26 PCB layers (13 double layers) are aligned within $10 \mu \mathrm{m}$.

In fact, a possible problem with the LDI machine is the misalignment of the top and bottom images, as seen for example in Fig. 6.

This may arise from errors in the panel fiducialization. To face this issue, mechanical fiducial markers were used instead of the usual optical targets. On each double layer, a matrix of nine fiducial holes was used at the four corners of each coil set to align the tracks on the top and the bottom faces. Additionally, larger fiducial 


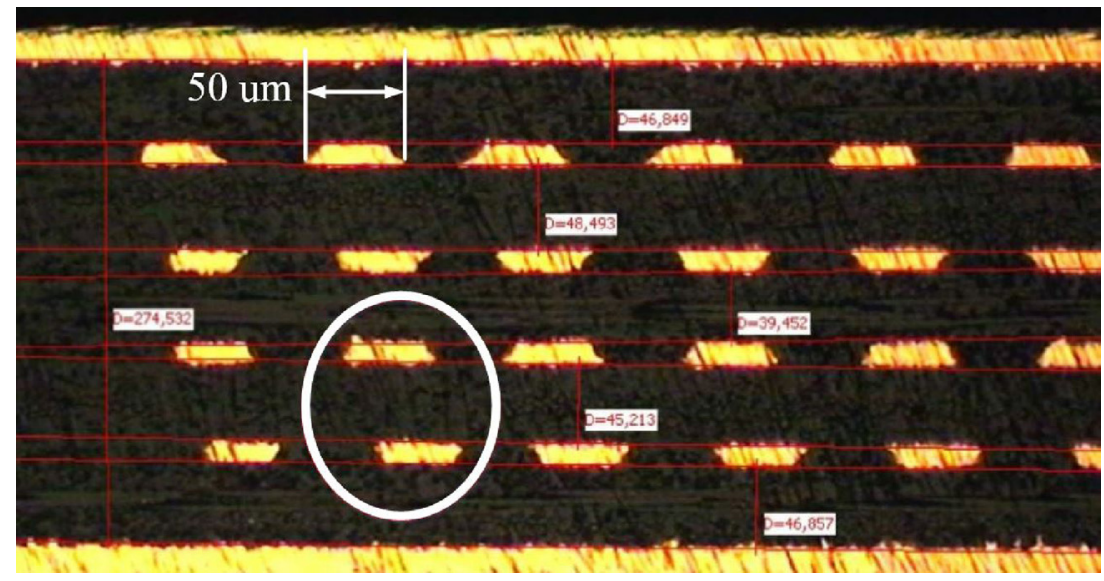

Fig. 6. Example of typical misalignment between tracks on top and bottom of the FR-4 layer.

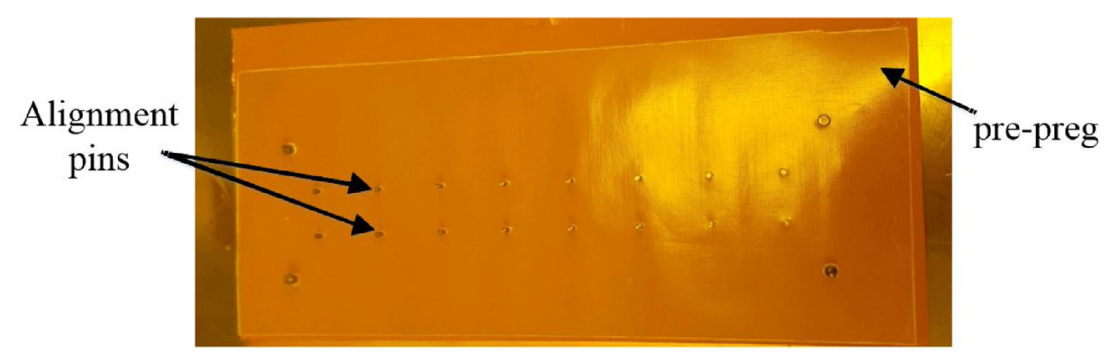

(a)

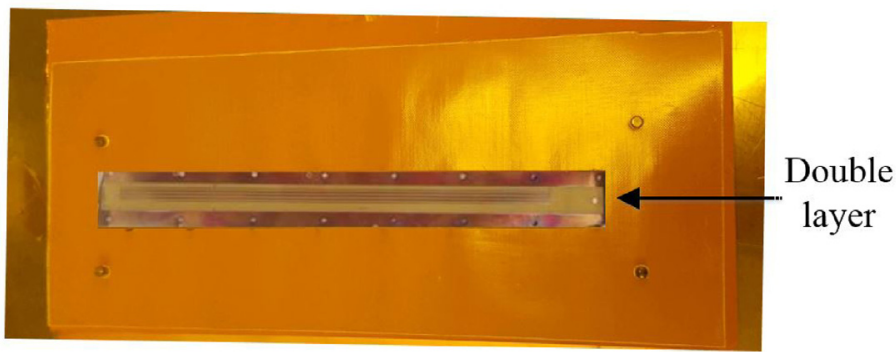

(b)

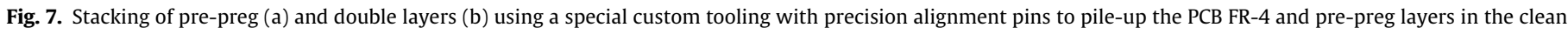
room.

holes were drilled next to the coils to align the double layers when stacked on top of each other (see Figs. 5 and 7).

The coupon, shown in Fig. 8, is the frame around the circuit that is cut away at the end of the PCB manufacturing process and that can be used to monitor its quality. This is done by adding in the coupon control tracks to be examined precisely at microscope.

Assembly of the double layers was verified with the help of special control tracks (Fig. 9), with increasing length, placed in correspondence of the board's edge. These control tracks are useful to verify aspects such as the correct stacking order of the double layers, the quality of the etching process, and the longitudinal misalignment.

Fig. 9 shows the double layer stacking coupon. The red windows in Fig. 9 highlight the area where the tracks are absent owing to a displacement induced when the board was cut. The problem was solved by enlarging the width of the edge control tracks.

The resistance and the inductance of the tracks on each layer were measured individually, before the final stacking, to verify the absence of inter-turn short-circuits (Fig. 7). For a given track crosssection, the resistance of a winding is proportional to the number of turns, while the inductance to the square. Therefore, the relative impact of a short that eliminates a small number of turns should be roughly double on the inductance than on the resistance: this provides a criterion to judge the consistency of the measurements.

The inductance was measured with the RLC-meter Agilent 4263Ba, equipped with micro-probes (due to the reduced size of vias holes and pads) at the frequency of $100 \mathrm{kHz}$. The average value measured for each single coil on a layer is $4.1 \mu \mathrm{H}$. The acceptance criterion was set at $1 \%$ of difference for the coils on the same layer and $5 \%$ for those on different layers. This takes into account that the inductance decreases logarithmically with the conductor crosssectional area, which in turn may be different from layer to layer, owing to variations in the etching process. About $50 \%$ of the production had to be rejected.

\section{Prototyping and quality control of the transducer}

\subsection{Shaft prototyping}

The shaft for the final version of the sensor (Fig. 10) is made of synthetic sapphire [32], manufactured by Insaco, Quakertown, PA, using optical-quality sapphire crystal, grown with the Kyropou- 


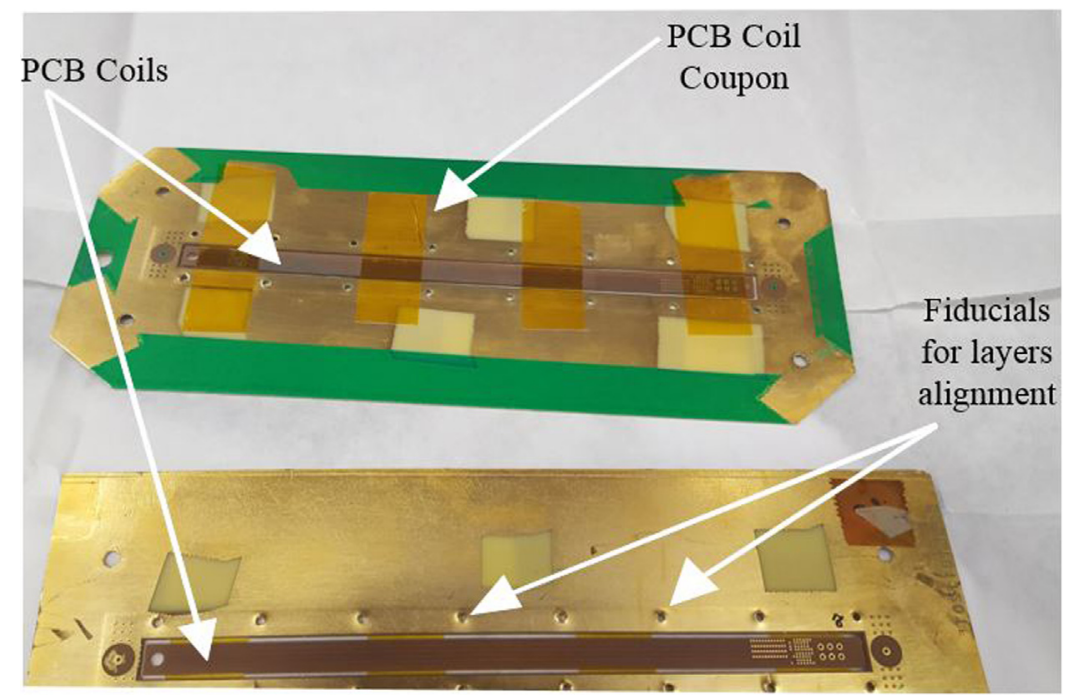

Fig. 8. The frame used as coupon board for microscope inspection and the miniaturized PCB coil.

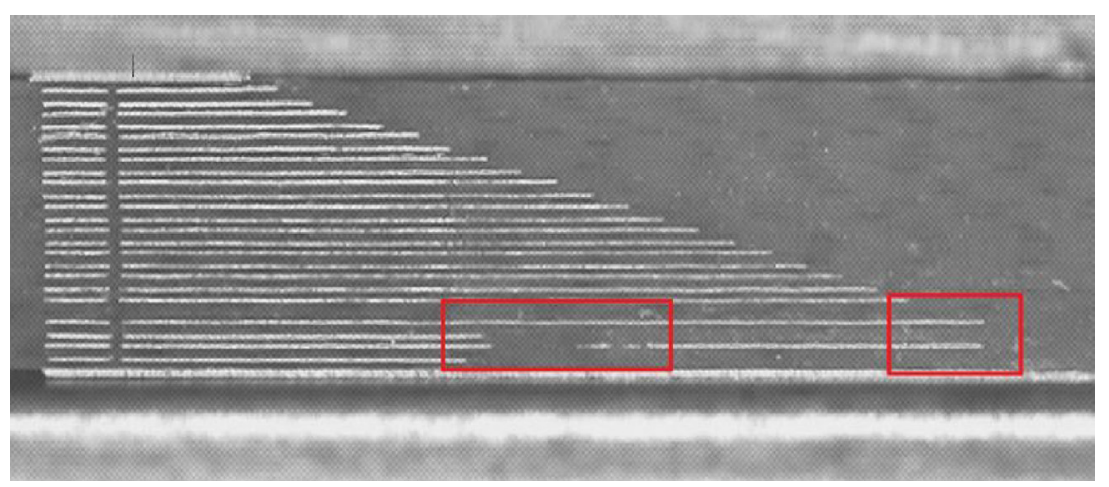

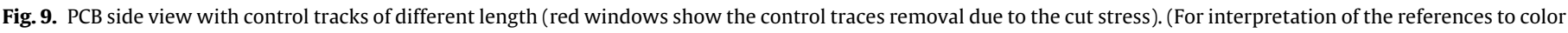
in this figure legend, the reader is referred to the web version of this article.)

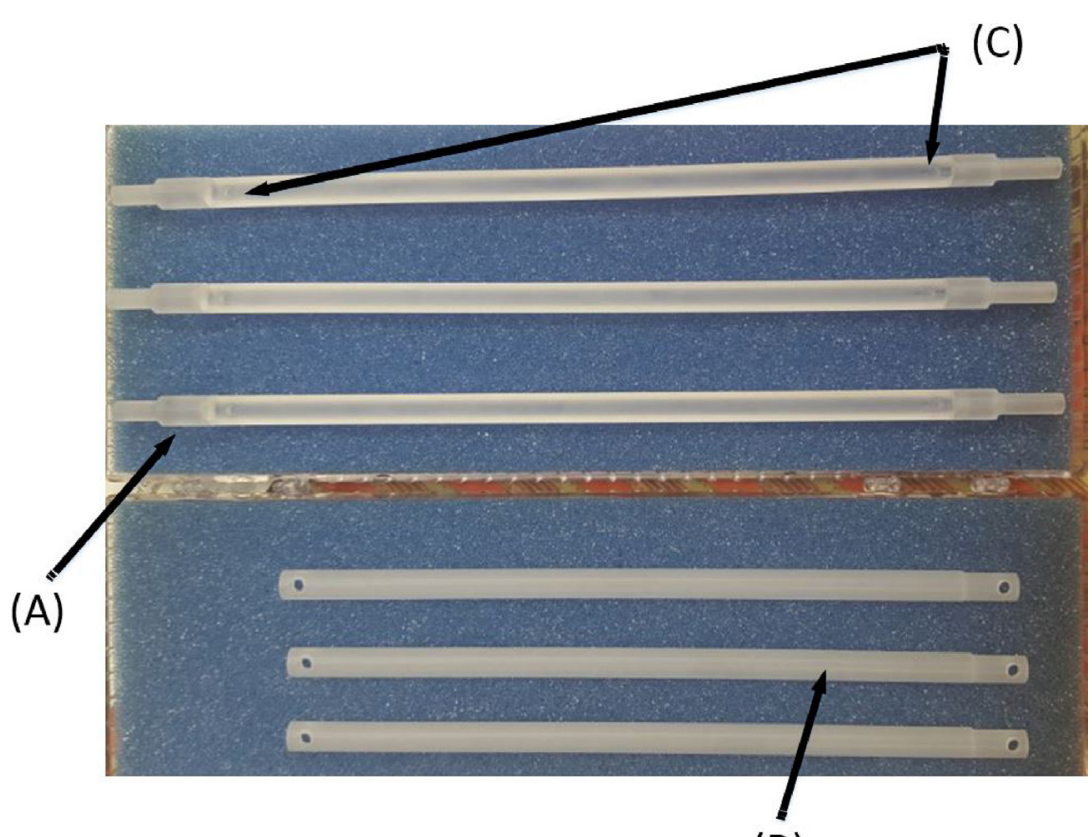

(B)

Fig. 10. $P C B$ sensor synthetic sapphire shafts: strong-back (A), cover (B), and alignment pins (C). 


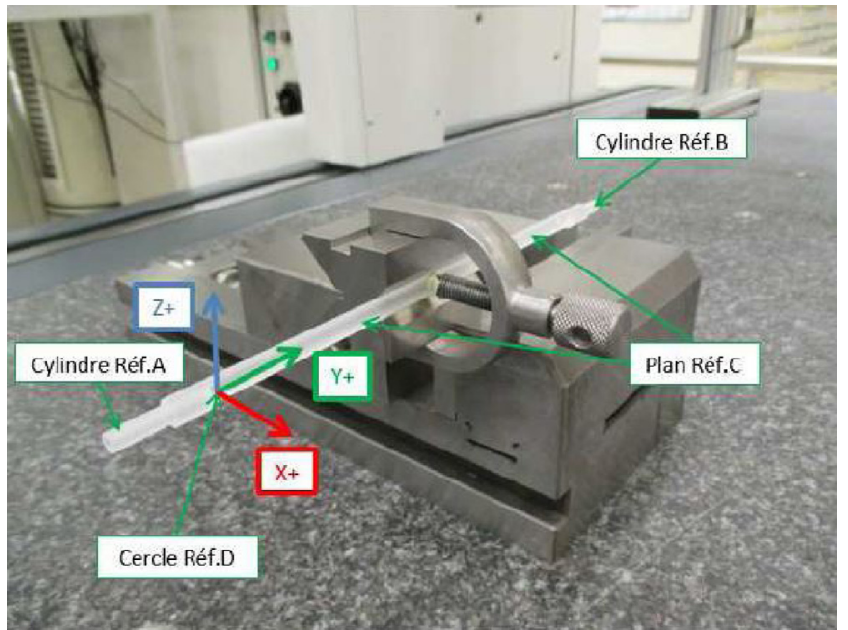

Fig. 11. Setup used by CERN metrology department to measure the synthetic sapphire shafts.

los technique [33]. Synthetic sapphire is made by sintering $\mathrm{Al}_{2} \mathrm{O}_{3}$ powder to a single-grain crystal and is non-magnetic and nonconducting, as required by the application. It is characterized by a very high Young's modulus ( $\approx 450 \mathrm{GPa})$, high manufacturing precision ( $5 \mu \mathrm{m}$ typical tolerance), and an extremely low surface roughness after polishing (typically down to $100 \mathrm{~nm}$ on a round surface or $50 \mathrm{~nm}$ on a flat surface). Synthetic sapphire has also an exceptionally high specific stiffness, about $110 \mathrm{MPam}^{3} \mathrm{~kg}^{-1}$, or about 8 times that of G10 fiberglass composite commonly used for the same purpose. This is the most important mechanical parameter for a rotating coil shaft, as it determines the amount of gravity-induced sag, with the consequent harmonic measurement errors [34]. The sensor shaft consist of three main parts 10: a strong-back (A), a cover (B), and a couple of alignment pins (c).

The PCB is aligned with the shaft using synthetic sapphire precision pins inserted in the strong-back holes. The blind holes are dimensioned for a touching probe with a spherical head of $2 \mathrm{~mm}$.

The shaft is supported by synthetic sapphire olive bearings at both ends to provide an extremely smooth rotation in order to reduce high-order harmonic errors [35]. In addition, their micrometer-level geometric tolerance, together with their special profile, allow to decouple the centering precision of the shaft for any small angular misalignment.

\subsection{Shaft quality control}

The geometry of the three produced shafts was measured by the CERN metrology service through the setup of Fig. 11.

Results show that the ends are concentric with an uncertainty between 1 and $3 \mu \mathrm{m}$, which is an order of magnitude better than fiberglass supports. Concentricity is particularly important because it affects the effective rotation radius of the coils. Uncertainty on this parameter, as it can be inferred from Eq. (9), will propagate exponentially with the harmonic order. Inspection has shown also that the pin holes used to mount the PCB coils are aligned with an uncertainty between 1 and $4 \mu \mathrm{m}$. The straightness of the shaft (measured with the method of the generating line) is between 5 and $8 \mu \mathrm{m}$.

These results show that synthetic sapphire shafts reach indeed a level of geometrical accuracy and mechanical performance very difficult to achieve with more traditional materials. The main drawback is represented by the high cost and by the extreme difficulty, using present-day technologies, to manufacture items longer than a few hundred millimeters, as would be required by several applications.

\section{Calibration and test results}

\subsection{Calibration}

The effectiveness of a compensated coil in suppressing a given field component $n$ is assessed by means of the so-called "bucking ratio" $B R_{n}$ [21]. They are defined by Eq. (12) in Appendix A.3, as the ratio between the absolute and compensated values of the $n$th harmonic component of the measured magnetic flux.

The bucking ratios for the dipole and quadrupole components of the two miniaturized PCB coils (mounted on the G10 and on the synthetic sapphire shaft) were measured in a reference permanent quadrupole at Fermilab. At this aim, the miniaturized sensor was inserted inside a provisional G10 shaft (Fig. 12).

The assembly was wrapped with adhesive kapton tape in order to retain a degree of flexibility.

The sensitivity factors $\kappa_{n}$ were calibrated with a permanent quadrupole magnet (Fig. 13), using the method described in [39].

This method uses DB and UB signals to calibrate the horizontal and vertical position of the PCB sensor inside the shaft. As a matter of fact, the quadrupole measured from the DB signal is independent of the radius, whilst the UB signal is radius dependent. The offset is calculated by comparing the modulus and phase of the UB and DB signals.

The modulus of nominal (uncalibrated) and calibrated normalized sensitivity values $\kappa_{n}^{*}$ are compared for the UB, DB, and DQB signals in Fig. 14, while in Fig. 15 the calibrated UB, DB and DQB signals are reported.

The design and actual board positions in the sapphire shaft differ owing to the manufacturing tolerances. In fact, the final PCB thickness $(1.28 \mathrm{~mm})$ is different from the design one $(1.38 \mathrm{~mm})$. Furthermore, the space left in the shaft was chosen by considering some tolerance and the adhesive tape. The results of calibrations underlines that the board was shifted of $-22 \mu \mathrm{m}$ radially and $-61 \mu \mathrm{m}$ horizontally. The PCB position can be shifted using adhesive precision layer of suitable thickness.

\subsection{Harmonic measurement results}

The measured harmonics of the Fermilab permanent quadrupole magnet are shown in Fig. 16: namely, normal on the left and skew on the right.

The 1-sigma repeatability of the normal (bn) and (an) skew harmonics is shown in Table 4: it turns out to be not greater than $0.20 \times 10^{-4}$ through $n=7$.

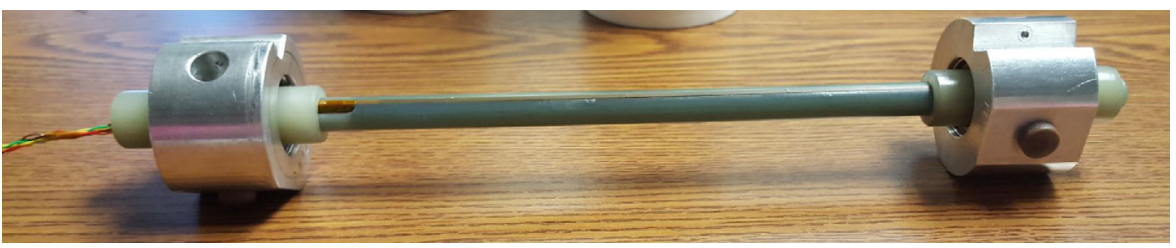

Fig. 12. PCB sensor with the $G 10$ shaft. 


\section{Bearing \\ holder}

\section{Synthetic}

Sapphire

rotating coil

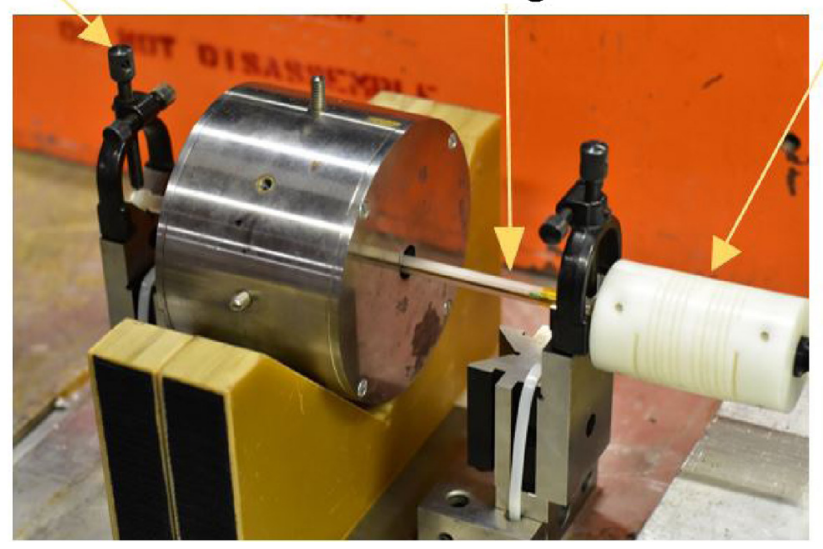

\section{Step-motor adaptation}

Fig. 13. Test-bench used at Fermilab to calibrate the synthetic sapphire rotating coil.
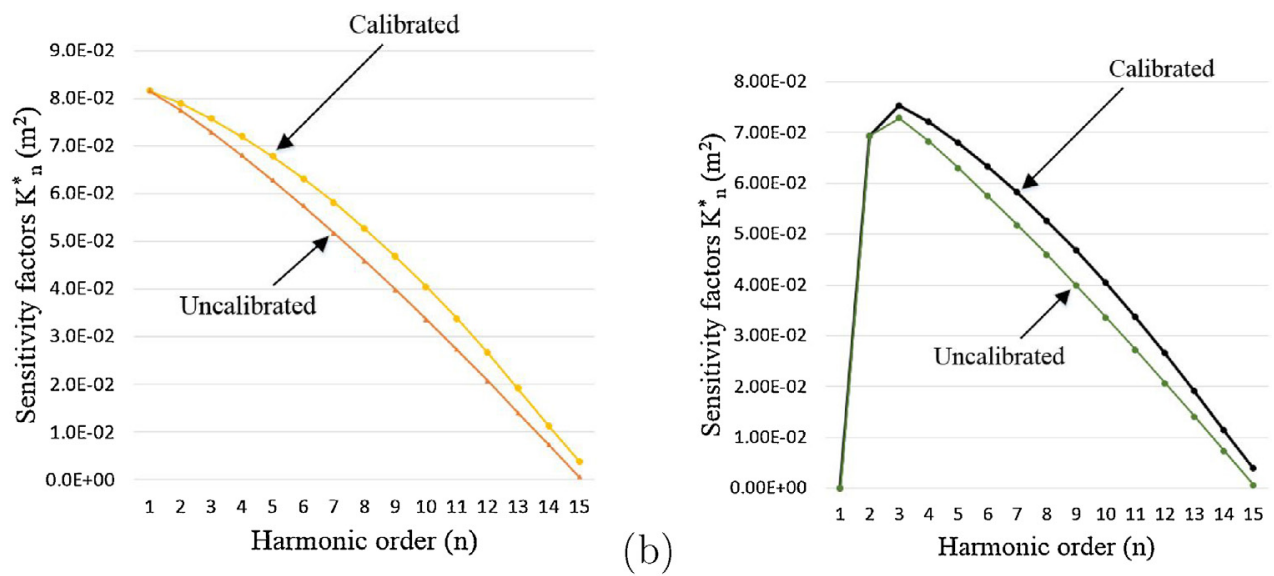

(a)

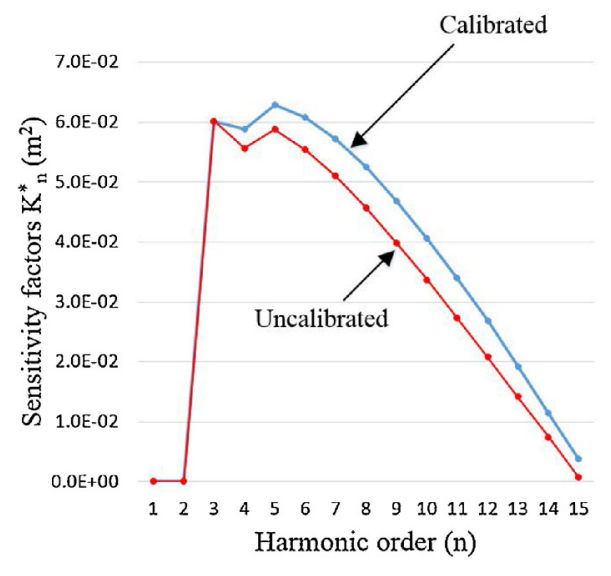

Fig. 14. Normalized sensitivity factors $\kappa_{n}^{*}$ before and after calibration of the signals: (a) unbucked (UB), (b) dipole-bucked (DB), and (c) dipole-quadrupole bucked (DBQ).

\section{Table 4}

1-Sigma repeatabiity on five turns of normal (bn) and skew (an) harmonics.

\begin{tabular}{llll}
\hline $\begin{array}{l}\text { Harmonic } \\
\mathrm{b}(\mathrm{n})\end{array}$ & $\begin{array}{l}\text { Standard deviation } \\
\left(10^{-4}\right)\end{array}$ & $\begin{array}{l}\text { Harmonic } \\
\mathrm{a}(\mathrm{n})\end{array}$ & $\begin{array}{l}\text { Standard deviation } \\
\left(10^{-4}\right)\end{array}$ \\
\hline 3 & 0.15 & 3 & 0.18 \\
4 & 0.18 & 4 & 0.07 \\
5 & 0.19 & 5 & 0.14 \\
6 & 0.17 & 6 & 0.10 \\
7 & 0.20 & 7 & 0.16 \\
\hline
\end{tabular}

The G-10 sensor version results, averaged over 10 consecutive turns, show a dipole bucking ratio of 120 in the DB signal and 138 in the DBQ, while the quadrupole bucking ratio is 106 . Despite the less-than-ideal initial mechanical setup, these results show an order-of-magnitude improvement with respect to the earlier sensors. The synthetic sapphire version has even better performance (Table 5). The dipole bucking ratio is 230 in the DB signal and 480 in the DQB, while the quadrupole bucking ratio 175. 


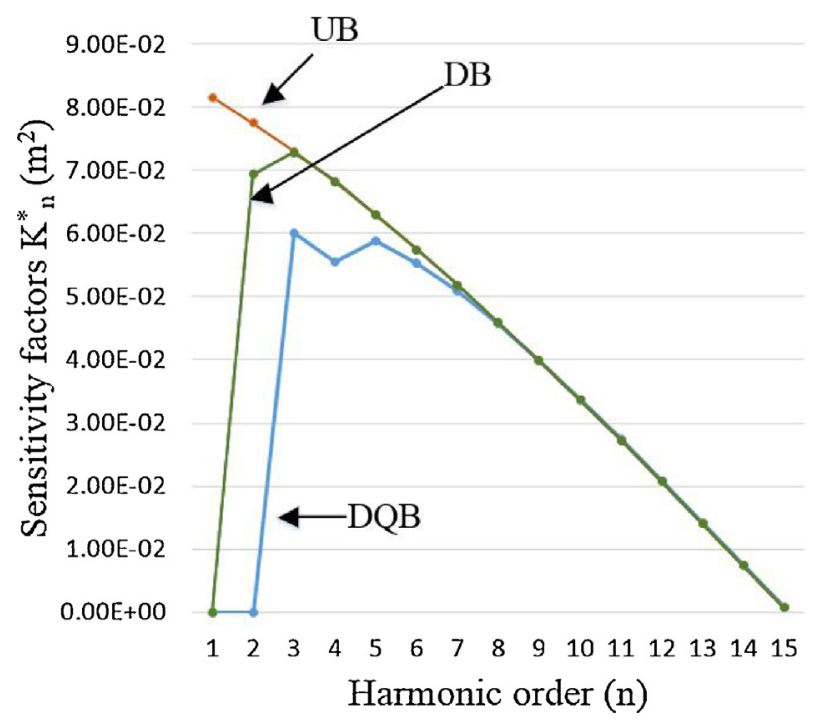

Fig. 15. Calibrated normalized sensitivity factors $\kappa_{n}^{*}$ of the signals: unbucked (UB, orange), dipole-bucked (DB, green), and dipole-quadrupole bucked (DBQ, blue). (For interpretation of the references to color in this figure legend, the reader is referred to the web version of this article.)

\section{Table 5}

Bucking ratios single-turn results and 1-sigma repeatability (DBR1: dipole bucking ratio of the DB signal, DBR2: dipole bucking ratio of DQB signal, and DQBR: quadrupole bucking ratio of DQB signal).

\begin{tabular}{llll}
\hline Turn & DBR1 & DBR2 & DQBR \\
\hline 1 & 260.8 & 420.8 & 175.3 \\
2 & 213.1 & 445.4 & 175.3 \\
3 & 208.6 & 426.9 & 174.0 \\
4 & 252.9 & 401.9 & 175.1 \\
5 & 278.9 & 476.8 & 175.2 \\
Mean & 242.9 & 434.4 & 175.0 \\
1-Sigma & 27.5 & 25.3 & 0.5 \\
\hline
\end{tabular}

The bucking ratios of the DB and DQB signals are stable within the tolerance in Table 2.

Currently, the same sapphire-based transducer is under test at Paul Scherrer Institute (PSI) in Switzerland for the planned crosscheck. Preliminary results show a quad bucking ratio over 1000: this points out that the Fermilab measurements of the bucking ratio presented here were affected by the magnet end fields, and performance of the proposed sensor could be significantly higher than expected.

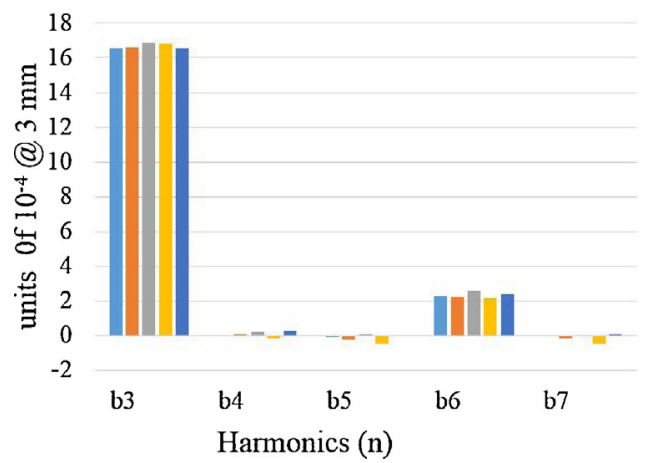

Table 6

Comparison between state-of-the-art mono-block PCB coil and new sapphire-based transducer coil transducers.

\begin{tabular}{lll}
\hline Parameter & Old PCB coil & New sapphire coil \\
\hline DQB bucking ratio & $<30$ & $>175$ \\
DB bucking ratio & - & $>400$ \\
On-board bucking & No & Yes \\
1-Sigma repeatability on harmonics & & \\
with amplitude less than $100 \mathrm{ppm}$ & $>5$ & $<0.5$ \\
Sensitivity up to harmonic order $(n)$ & 4 & 8 \\
Possibility to modify coils radii & No & Yes
\end{tabular}

\subsection{Comparison with state-of-the-art mono-block $P C B$ coil transducer}

The state-of-the-art mono-block PCB coil [25] and the new sapphire-based transducer coil transducers are compared in Table 6.

With respect to the state-of-the-art transducer, the sapphirebased transducer has better metrological performance, namely (i) more than five times better accuracy, arising from on-board harmonic error compensation (higher Dipole quadrupole bucking ratio $>175$ ), and (ii) 1-sigma repeatability ten times better on harmonics with amplitude less than $100 \mathrm{ppm}$.

Furthermore, the sapphire-based transducer is designed with embedded compensation, with three output bucking signals, UB, $\mathrm{DB}$, and DQB. The DB signal is radius independent and thus is suitable to be calibrated by the Fermilab procedure.

The state-of-the-art transducer has an inferior DQB ratio $(<30$ for the best case [37]) is not designed for on-board bucking, and the relative position of coils inside the shaft cannot be modified after manufacturing.

The sapphire-based transducer has a maximum uncertainty on relative coils position below $<5 \mu \mathrm{m}$, while the state-of-the-art transducer had a maximum uncertainty from $65 \mu \mathrm{m}$ [37] up to $160 \mu \mathrm{m}$ [25]. Finally, the position of the four PCB assemblies inside the synthetic sapphire can be adjusted opportunely after the manufacturing using layers of precision epoxy.

\section{Conclusions}

An innovative, ultra-thin (less than $2 \mathrm{~mm}$ ) coil array, with a compensation scheme for the main field components, jointly developed by CERN and Fermilab, has been presented. In this very-small size range, manufacturing technology and metrological quality control of both the array sensor and the transducer turn out to be beyond the state of the art of magnetic measurements. The problem of harmonic coils thickness with unacceptable sensitivity loss to some harmonics for small magnet gaps (when the ratio of the thickness to the rotation radius exceeds a few percent) has been success-

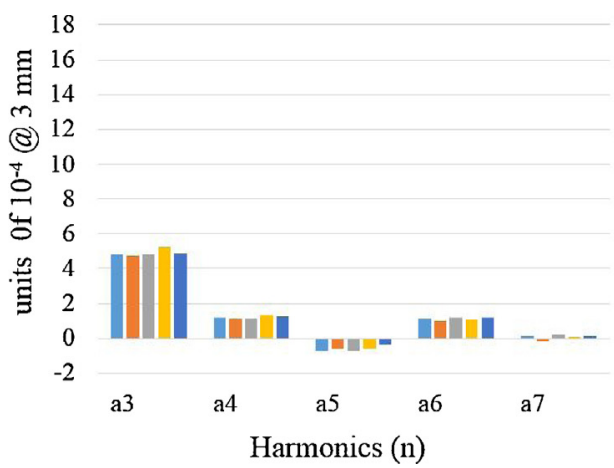

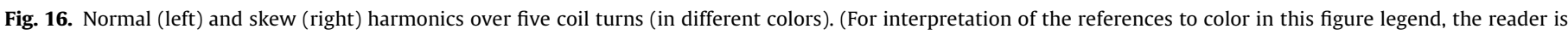
referred to the web version of this article.) 
fully overcome by the proposed sensor. This was proved in extreme cases, such as the PCB coil for $10 \mathrm{~mm}$ diameter of circular bore of CLIC quadrupoles discussed in this paper. A practical way to do so has been successfully demonstrated by pushing the envelope of widely available PCB fabrication techniques.

Innovation arises from both the design and the manufacturing: (i) very-small size, both for the sensing coil array (thickness of $1,380 \mathrm{~mm}$ ) and for the transducer as a whole (overall diameter of $7.350 \mathrm{~mm}$ ), (ii) metrological performance, namely accuracy (more than five times better than state of the art), arising from on-board harmonic error compensation, and 1-sigma repeatability (ten times better than state of the art on harmonics with amplitude less than $100 \mathrm{ppm}$ ), (iii) and manufacturing technology and metrological quality control of both the PCB coil array (13 double layers are aligned within $10 \mu \mathrm{m}$ ), and the synthetic sapphire support (for concentricity, the most important uncertainty source for rotating coils transducers, $3 \mu \mathrm{m}$ of uncertainty, namely one order of magnitude better than fiberglass). The most striking improvement concerns the bucking factor of the dipole and quadrupole components, which has been improved by more than two orders of magnitude with respect to more traditional designs.

This issue is expected to gain more importance in future because of the trend towards smaller magnet apertures and higher field quality in the domain of linear particle accelerators, both for physical research or for medical purposes. Further development of compensated PCB coil arrays, especially focused on innovative coil support materials and longer coils, is planned in the context of a wider collaboration between CERN, FermiLab, and PSI. Future actions include the evaluation of systematic calibration errors via the comparison of measurements carried out with the new PCB coil and other instruments, such as other coils of the same type and a vibrating wire, on a suitable reference magnet.

\section{Acknowledgements}

The work presented was funded by the project PACMAN [20], an action "Marie Curie" within the FP7 Framework Program of the European Union. This project, led by Helene Mainaud Durand (CERN), involves the collaboration between CERN and a large network of universities and industrial partners.

The Authors thank Stephan Russenschuck, Michele Modena, Francois Nicolas Morel, Serge Ferry and Bertrand Mehl (CERN) and Dan Assell, George Velev, with all the FermiLab team, for technical and scientific support. The Authors thank the Anonymous Reviewers for the useful suggestions that improved significantly the readability of the paper.

\section{Appendix A. The harmonic coil method}

\section{A.1 Basic theory}

The magnetic field generated by a typical cylindrical accelerator magnet, when integrated along its longitudinal axis (corresponding to the beam path), satisfies the 2D Laplace equation on a transverse section. Therefore, it can be described by the complex harmonic expansion [36]:

$B(z)=B_{y}+i B_{x}=\sum_{n=1}^{\infty} C_{n}\left(\frac{z}{R_{\text {ref }}}\right)^{n-1}$

where $C_{n}=B_{n}+i A_{n}$ is the $n$th complex harmonic coefficient, $B_{n}$ and $A_{n}$ the normal and skew field components, respectively, and $R_{r e f}$ a reference radius, which usually approximates the maximum beam size.

In most practical cases, magnets are designed to provide only one main harmonic component $n$, typically with $1 \leq n \leq 6$. For $n \geq 2$, harmonics of order smaller than $n$ depend on the position of the origin of the coordinate system ("feed-down effect" [12]). The magnetic axis is the locus where magnetic field is null by definition. For a quadrupole magnet $(n=2)$, when a coordinate axis coincides with the magnetic axis, $a_{1}$ and $b_{1}$ vanish; while if $n=3$, the nullity of $a_{2}$ and $b_{2}$ can be taken as definition of magnetic axis ( $a_{1}$ and $b_{1}$ may not vanish). Higher harmonics, commonly due to construction and material imperfections, tend to decrease as $R_{\text {ref }}^{-n}$ for $n \rightarrow \infty$ (based on the Cauchy estimate for holomorphic functions) [12].

The radial $B_{r}$ and tangential $B_{t}$ field components (Fig. 17) can be

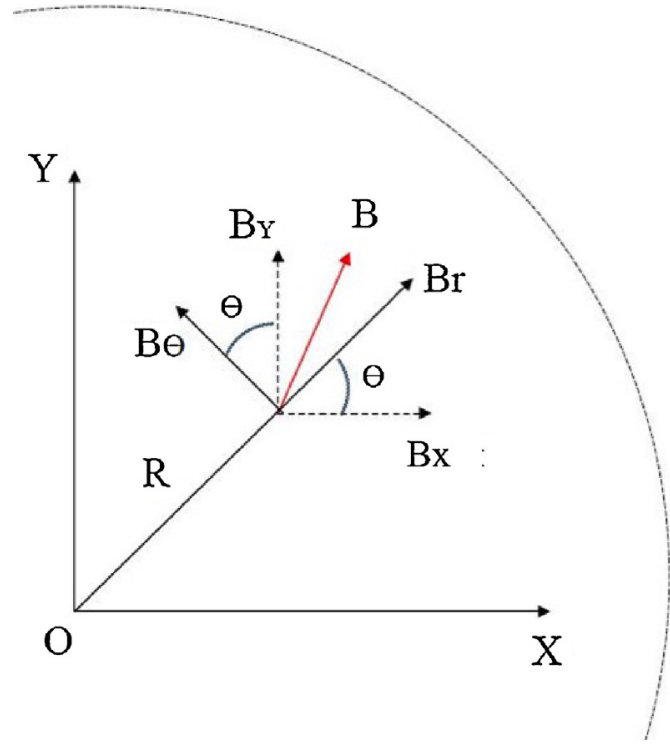

Fig. 17. Field components frame in magnet bore.

expressed in polar coordinates:

$B_{r}=\operatorname{Im}\left(B e^{i \theta}\right)=\sum_{n=1}^{\infty}\left(\frac{r}{R_{\text {ref }}}\right)^{n-1}\left[A_{n} \cos (n \theta)+B_{n} \sin (n \theta)\right]$
$B_{t}=\operatorname{Re}\left(B e^{i \theta}\right)=\sum_{n=1}^{\infty}\left(\frac{r}{R_{\text {ref }}}\right)^{n-1}\left[B_{n} \cos (n \theta)-A_{n} \sin (n \theta)\right]$

These components contain exactly the same information in terms of their Fourier coefficients; therefore, in principle they can be chosen as measurands in order to reconstruct the field as a whole.

\section{A.2 Coil geometry}

The coils used to measure the integral field of an accelerator magnet are usually long rectangular loops of wire, constrained in width by the relatively-narrow magnet aperture, mounted on a support shaft that allows rotation around a longitudinal axis. The geometry of an ideal coil can be described by the complex points $z_{1}$ and $z_{2}$, as shown in cross-section (Fig. 18), representing the location of the conductor turns in the transverse plane. The origin can be assumed as coincident with the trace on the section of the rotation axis, not necessarily coinciding with the magnetic axis of the magnet under test. In case of a multiple winding, all the turns can be assumed as lumped together as the central positions in the analysis, with negligible uncertainty [38].

The position of the windings during the rotation can be expressed as:

$z_{1}(\theta)=z_{1,0} e^{i \theta}=R_{1} e^{i\left(+\frac{\Delta \theta}{2}+\theta-\theta_{0}\right)}$ 


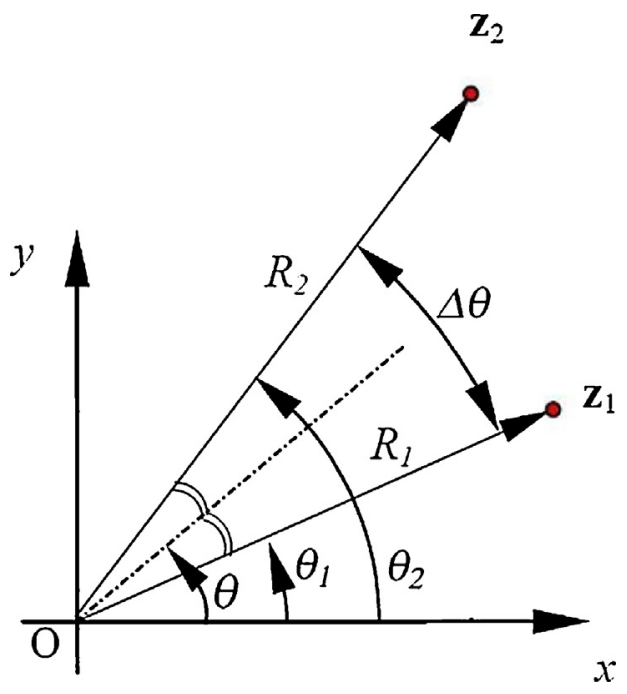

Fig. 18. Filament location in the complex plane.

$z_{2}(\theta)=z_{2,0} e^{i \theta}=R_{2} e^{i\left(-\frac{\Delta \theta}{2}+\theta-\theta_{0}\right)}$

where the index 0 denotes the initial position of the windings and $\theta_{0}$ the initial phase of the coil. Usually, $\theta_{0}$ is defined by the bisector of the coil opening angle $\Delta \theta$ (Fig. 17), when an angular encoder outputs a reference trigger pulse.

The magnetic flux linked through the coil during its rotation at an arbitrary angle is given by the real part of the complex magnetic potential:

$\Phi(\theta)=N_{t} L_{c} \operatorname{Re} \int_{z_{1}(\theta)}^{z_{2}(\theta)} B d z=\operatorname{Re} \sum_{n=1}^{\infty} \frac{\kappa_{n}}{R_{r e f}^{n-1}} C_{n} e^{i n\left(\theta-\theta_{0}\right)}$

where $N_{t}$ is the number of winding turns, $L_{c}$ the axial length of the coil, and $\kappa_{n}$ the coil sensitivity coefficients:

$\kappa_{n}=\frac{N_{t} L_{c}}{n}\left(z_{2,0}^{n}-z_{1,0}^{n}\right)=\frac{N_{t} L_{c}}{n}\left(R_{2}^{n} e^{i n \frac{\Delta \theta}{2}}-R_{1}^{n} e^{-i n \frac{\Delta \theta}{2}}\right)$

When the windings size invalidates the lumped approximation, this expression can be generalized by using a sum over individual half-turns [39]:

$\kappa_{n}=\frac{N_{t} L_{c}}{n} \sum_{j=1}^{2 N_{t}} z_{j, 0}^{n}(-1)^{j}$

where $z_{j, 0}$ represents the complex coordinates of the $j$ th single halfturn filament in the initial position, ordered so that the out-going and return halves of the wire loops alternate. This formulation is particularly useful for PCB-based coils, where the coordinates of all individual conductor tracks are available by design.

The $\kappa_{n}$ have the physical units of meters to power $(n+1)$ and a magnitude varying exponentially with $n$. In the following, they are normalized with respect to the reference radius in order to have all the dimension of an area $\left(\mathrm{m}^{2}\right)$ and a comparable order of magnitude:

$\kappa_{n}^{*}=\frac{\kappa_{n}}{R_{r e f}^{n-1}}$

The $\kappa_{n}$ depend only upon the geometry of the coil and can be computed by calibration through the coil area, the rotation radius, and the tilt angle. These three scalar parameters, together with the initial phase $\theta_{0}$, are geometrically equivalent to the two complex numbers $z_{10}$ and $z_{20}$. The initial phase depends upon the way the coil is mechanically mounted with respect to the reference frame (provided by the encoder), and therefore is a property of the transducer as a whole, rather than of the coil alone.

While, in principle, sensing coils can be built with any arbitrary placement of windings $z_{1,0}$ and $z_{2,0}$, the two most used geometries are radial (where the phase is the same) and tangential [12]. In accordance with the divergence of the magnetic field being zero in a source-free region, the radial and the tangential field intercepted by a tangential or a radial coil, respectively, contains the same information. The choice between the two options depends upon many practical factors, such as the mechanical design of the rotating shaft and the available space. As a main criterion, since for harmonic order $n$, signal levels increase with $R^{n-1}$. Thus, the largest signal on higher orders will be given by a coil mounted tangentially on a circular shaft with the largest diameter fitting the aperture. This advantage, however, is balanced by the difficulty to achieve tight positioning tolerances by a tangential configuration and, more importantly, by some sensitivity terms approaching zero in (7), interpreted as a continuous function of $n$ :

$\kappa_{n}=0 \Rightarrow e^{i n \frac{\Delta \theta}{2}}-e^{-i n \frac{\Delta \theta}{2}}=0 \quad\left(R_{2}=R_{1}\right)$

This is intuitively evident if a coil with an opening angle $\Delta \theta$ exactly equal to a sub-multiple of $2 \pi$ is considered:

$\Delta \theta=\frac{2 \pi}{m} \Rightarrow \kappa_{2 j m}=0, j=1,2, \ldots$

Such a coil will not link any flux of the corresponding $2 \mathrm{jm}$ field harmonic (and all of its integer multiples) at any angle during the rotation. The zeros will fall between two harmonics, causing the magnitude of the corresponding $\kappa_{n}$ to be very small. This loss of information in the measured flux can be minimized by designing a tangential coil so that the zeros fall exactly in the middle between two harmonics. In general, Eq. (7) cannot have zeros if the radii $R_{1}$ and $R_{2}$ are different.

\section{A.3 Harmonic compensation}

Mechanical imperfections (due to assembly errors) and rotation errors (vibration and torsion) degrade metrological performance of a single rotating coil measuring high-order harmonics, even if ideally designed for measuring all the harmonic content of a magnet. In particular, torsional or flexural vibrations (rotation errors) of the coil give rise to spurious harmonic content, roughly proportional to the dominant order or to the one immediately below, respectively [34]. Mechanical perturbations cannot be made negligible; therefore, a further coil, ideally insensitive to these two components, is added. This is commonly referred to as harmonic compensation (or colloquially bucking). A coil measuring the main field component, instead, is referred to as absolute.

In practice, compensation is easily achieved by taking a suitable linear combination of the outputs of two or more individual coils, arranged in a radial or tangential array. The design and the construction of such an array are simpler when all the coils are equal and uniformly spaced, although this is by no means necessary.

The compensation can be of two types:

- analogue: the coils are physically connected in series or series opposition, if possible via an easy reconfiguration of the setup. Hard-wired connections, especially practical for arrays on the same PCB, offer higher reliability. In both cases, common mode perturbations are rejected, thus providing a higher signal-tonoise ratio. The compensation effectiveness depends critically on the manufacturing quality of the coils; in particular, for arrays with equal nominal areas of the single coil, tolerance should not exceed a few tens of ppm. With standard winding, such a tight 
requirement can be met by fabricating and sorting more units than actually necessary (at least three times as many).

- digital: the signals of all coils of the array are acquired individually and then compensated numerically. This method offers flexibility, because the signals can be scaled and phase-shifted at will, by using complex coefficients in the linear combination. This not only increases vastly the range of possible compensation schemes, extending them to multiple harmonic orders with the same physical array, but also allows compensation of coil construction and assembly errors, leading to different coil surfaces or angular mounting errors. Drawbacks include higher cost of multi-channel acquisition and the related uncertainty, due to mismatch and drift of gain, as well as noise limitations and offset of individual acquisition channels.

The effectiveness of a compensated coil in suppressing a given field component $n$ can be assessed by means of the so-called "bucking ratio" [21]:

$\mathrm{BR}_{n}=\frac{\Psi_{n}^{\mathrm{abs}}}{\Psi_{n}^{\mathrm{cmp}}}$

where $\Psi_{n}$ represents the $n$th harmonic component of the measured magnetic flux and the superscripts $a b s$ and $\mathrm{cmp}$ denote the absolute and compensated values, respectively. Often, the absolute coil is a part of the array used for the compensation, typically the outermost coil as it will output the largest signal in a multipole. In this case, the bucking ratio provides a consistent comparison between intrinsically the same quantity, with and without the unwanted harmonics. $B R_{n}$ depends both on the coil geometry and on the signal acquired during a specific measurement; therefore, its measured value in repeated measurements is affected by electrical and mechanical uncertainty.

$B R_{n}$ tends to infinity when the compensation is perfect, i.e. when the $n$th harmonic component vanishes from the measured flux. By traditional winding techniques, bucking factors of the order of a few 100 's, for the dipole, and 50-100 for the quadrupole, are commonplace. The phase, on the other hand, contains information on the construction quality of the coil array (e.g., in case of two equal coils connected in opposition to cancel out the dipole, but having a small parallelism error).

\section{References}

[1] P. Ripka, Advances in fluxgate sensors, Sens. Actuators A: Phys. 106 (1) (2003) 8-14

[2] W. Li, S. Liao, C. Tsou, A novel sensing chip with dual-coil inductance for determining raw milk quality, Sens. Actuators A: Phys. 241 (2016) 96-103.

[3] M. Yamaguchi, S. Yabukami, K.I. Arai, Development of multiplayer planar flux sensing coil and its application to $1 \mathrm{MHz}-3.5 \mathrm{GHz}$ thin film permeance meter, Sens. Actuators 81 (2000) 212-215.

[4] D. Roetenberg, P. Slycke, A. Ventevogel, P.H. Veltink, A portable magnetic position and orientation tracker, Sens. Actuators A: Phys. 135 (2) (2007) 426-432.

[5] H. Can, P. Svec, J. Bydzovsky, H. Sozeri, U. Topal, Systematic optimization of the sensing properties of ring-core fluxgate sensors with different core diameters and materials, Sens. Actuators A: Phys. 255 (2017) 94-103.

[6] E. Paperno, A. Plotkin, Cylindrical induction coil to accurately imitate the ideal magnetic dipole, Sens. Actuators A: Phys. 112 (2) (2004) 248-252.

[7] P. Brauer, T. Risbo, J.M. Merayo, O.V. Nielsen, Fluxgate sensor for the vector magnetometer onboard theAstrid-2'satellite, Sens. Actuators A: Phys. 81 (1) (2000) 184-188.

[8] T.M. Liakopoulos, C.H. Ahn, A micro-fluxgate magnetic sensor using micromachined planar solenoid coils, Sens. Actuators A: Phys. 77 (1) (1999) 66-72.

[9] A.W. Chao, K.H. Mess, M. Tigner, F. Zimmermann (Eds.), Handbook of Accelerator Physics and Engineering, World Scientific, 2013.

[10] P. Schnizer, A. Mierau, A. Bleile, V. Marousov, A. Stafiniak, W. Freisleben, H. Mueller, Low-temperature test capabilities for the superconducting magnets of FAIR, IEEE Trans. Appl. Supercond. 25 (3) (2015) 1-5.
[11] N. Sammut, L. Bottura, G. Deferne, W.V. Delsolaro, Mathematical formulation to predict the harmonics of the superconducting Large Hadron Collider magnets: III. Precycle ramp rate effects and magnet characterization, Phys. Rev. Spl. Top.-Acceler. Beams 12 (10) (2009) 102401.

[12] S. Russenschuck, Field Computation for Accelerator Magnets: Analytical and Numerical Methods for Electromagnetic Design and Optimization, Wiley, 2011.

[13] L. Fiscarelli, B. Auchmann, S.I. Bermudez, B. Bordini, O. Dunkel, M. Karppinen, G. Willering, Magnetic measurements and analysis of the first 11-T Nb $3 \mathrm{Sn}$ dipole models developed at CERN for HL-LHC, IEEE Trans. Appl. Supercond. 26 (4) (2016) 1-5

[14] G. Dehmel, Magnetic field sensors: induction coil (search coil) sensors. Sensors - A Comprehensive Survey, vol. 5, VCH, New York, 1989, pp. 205-254 (Chapter 6).

[15] S. Tumanski, Induction coil sensors - a review, Meas. Sci. Technol. 18 (3) (2007) R31.

[16] P. Ripka, Induction sensors. Magnetic Sensors and Magnetometers, Artech House, Boston, MA, 2001, pp. 47-74 (Chapter 2).

[17] A. Alfadhel, A.A.A. Carreno, I.G. Foulds, J. Kosel, Three-axis magnetic field induction sensor realized on buckled cantilever plate, IEEE Trans. Magn. 49 (7) (2013) 4144-4147.

[18] O. Le Contel, P. Leroy, A. Roux, C. Coillot, D. Alison, A. Bouabdellah, R.B. Torbert, The search-coil magnetometer for MMS, Space Sci. Rev. 199 (1-4) (2016) 257-282.

[19] J. Campmany, J. Marcos, V. Massana, New improvements in magnetic measurements laboratory of the ALBA synchrotron facility, Phys. Proc. 75 (2015) 1214-1221.

[20] https://pacman.web.cern.ch.

[21] M. Buzio, Manufacturing and Calibration of Search Coils, CAS on Magnet, Bruges, 2009, ISBN: 9789290833550.

[22] O. Köster, L. Fiscarelli, S. Russenschuck, A procedure for combining rotating-coil measurements of large-aperture accelerator magnets, Nucl. Instrum. Methods Phys. Res. Sect. A: Acceler. Spectrom. Detect. Assoc. Equip. 818 (2016) 20-25.

[23] J. Billan, L. Bottura, M. Buzio, G. D’Angelo, G. Deferne, O. Dunkel, S. Schloss, Twin rotating coils for cold magnetic measurements of $15 \mathrm{~m}$ long LHC dipoles, IEEE Trans. Appl. Supercond. 10 (1) (2000) 1422-1426.

[24] Z. Ang, I. Bejar, L. Bottura, D. Richter, M. Sheahan, L. Walckiers, R. Wolf, Measurement of AC loss and magnetic field during ramps in the LHC model dipoles, IEEE Trans. Appl. Supercond. 9 (2) (1999) 742-745.

[25] H. Braun, M. Buzio, R. Deckardt, O. Dunkel, R. Felder, L. Fiscarelli, R. Ganter, S. Kasaei, F. Löhl, S. Sanfilippo, G. Severino, V. Vrankovic, L. Walckiers, Small-Diameter Rotating Coils For Field Quality Measurements in Quadrupole Magnets, IMEKO14, Benevento, Italy, 2014, September.

[26] M. Buzio, G. Golluccio, A. Lombardi, F. Mateo, Magnetic qualification of permanent magnet quadrupoles for CERN's Linac4, in: International Conference on Magnet Technology MT22, Marseille, 2011.

[27] H. Mainaud Durand, K. Artoos, M. Buzio, D. Caiazza, N. Catalan Lasheras, A. Cherif, I. Doytchinov, A. Faus-Golfe1, J.-F. Fuchs, A. Gaddi, N. Galindo Munoz, J.-C. Gayde, S.W. Kamugasa, M. Modena, P. Novotny, S. Russenschuck, C. Sanz, G. Severino, D. Tshilumba, V. Vlachakis, S. Zorzetti, Main Achievements of the PACMAN Project for the Alignment at Micrometric Scale of Accelerator Components, IPAC17, Copenhagen, Denmark, 2017, May.

[28] M. Modena, D. Tommasini, A. Vorozhtsov, Design and manufacture of a hybrid final focus quadrupole model for CLIC, IEEE Trans. Appl. Supercond. 22 (2012).

[29] J. DiMarco, Application of PCB and FDM Technologies to Magnetic Measurement Probe System Development, Prentice Hall, New Jersey, 1999.

[30] J. DiMarco, G. Chlachidze, A. Makulski, D. Orris, M. Tartaglia, J.C. Tompkins, X. Wang, Application of PCB and FDM technologies to magnetic measurement probe system development, IEEE Trans. Appl. Supercond. 23 (3) (2013) 9000505.

[31] M. Bajko, M. Buzio, et al., Geometric and magnetic axes of the LHC dipole, in: Proceedings of the Particle Accelerator Conference, Chicago, 2001

[32] M. Bickermann, Sapphire Crystal Growth, University Erlangen-Nürnberg, Erlangen, Germany, https://www.ikz-berlin.de/bickermann/uni/silberbach// STU08S.pdf.

[33] W.A. Bonner, L.G. Van Uitert, Growth of single crystals of Pb 3 MgNb 2 O 9 by the Kyropoulos technique, Mater. Res. Bull. 2 (1) (1967) 131-134.

[34] A. Jain, Measurement and Alignment of Accelerator and Detector Magnets, 11-17 April 1997, CAS - CERN Accelerator School, Anacapri, Italy, 1998, pp. 175-218 (CERN-1998-005), 10.5170/CERN-005.175.

[35] C.M. Spencer, S.D. Anderson, D.R. Jensen, Z.R. Wolf, A rotating coil apparatus with sub-micrometer magnetic center measurement stability, IEEE Trans. Appl. Supercond. 16 (2) (2006) 1334-1337.

[36] L. Walckiers, Magnetic Measurement With Coils and Wires, CERN-2010-004, pp. 357-385, arXiv:1104.3784 [physics.acc-ph].

[37] O. Dunkel, A Rotating Coil Array in "Mono-Bloc" Printed Circuit Technology for Small Scale Harmonic Measurements, IMMW 17, 18-23 September, Terrassa-Barcelona, Catalonia, Spain, 2011.

[38] L. Deniau, Coils Calibration Correction Factor for Rectangular Windings, CERN, MTA-IN-98-026, https://edms.cern.ch/document/356512/1.

[39] J. DiMarco, Application of PCB and FDM Technologies to Magnetic Measurement and Probe Development, IMMW18, June, 2013. 


\section{Biography}

P. Arpaia took Master Degree and PhD in Electrical Engineering at University of Napoli Federico II (Italy), where he is professor of Instrumentation and Measurements. He is Team Leader at European Organization for Nuclear Research (CERN). He was also professor at University of Sannio, scientific associate at Institutes of Engines and Biomedical Engineering of CNR, and now of INFN Section of Naples.

He is Associate Editor of the Elsevier Journal Computer Standards \& Interfaces, and in the past also of IEEE Transactions on Electronics Packaging and Manufacturing. $\mathrm{He}$ is Editor at Momentum Press of the Book Collection "Emerging Technologies in Measurements, Instrumentation, and Sensors". In last years, he was scientific respon- sible of more than 30 awarded research projects in cooperation with industry, with related patents and international licences, and funded 4 academic spin off companies. He acted as scientific evaluator in several international research call panels. He has served as organizing and scientific committee member in several IEEE and IMEKO Conferences. He is plenary speaker in several scientific conferences.

His main research interests include digital instrumentation and measurement techniques for magnets, superconductors, power converters and cryogenics of particle accelerators, evolutionary diagnostics, distributed measurement systems, ADC modelling and testing. In these fields, he published several book chapters, and about 235 scientific papers in journals and national and international conference proceedings. His PhD students were awarded in 2006 and 2010 at IEEE I2MTC, as well as in 2012 and 2016 at IMEKO World and TC-10 Conferences, respectively. 